Rapid Reviews COVID-19

\title{
Review 2: "Data From the \\ COVID-19 Epidemic in \\ Florida Suggest That \\ Younger Cohorts Have \\ Been Transmitting Their \\ Infections to Less Socially \\ Mobile Older Adults"
}

Lee Worden ${ }^{1}$

1UCSF

Published on: Aug 11, 2020

DOI: 10.21428/2e3983f5.4956c5a3

License: Creative Commons Attribution 4.0 International License (CC-BY 4.0). 
[Note from Editor: This review has chosen to answer select questions from the $\underline{R R: C 19}$ peer review guide that is suggested to reviewers, followed by detailed comments aimed at improving the author's manuscript.]

Are the findings novel?

Yes, in that this paper reports new results from the state of Florida. Similar results appear to have been reported based on data from other locations (e.g. Yu, 2020, https://doi.org/10.1101/2020.05.17.20105049).

Are the findings "interesting" in that they contribute to broader research understandings or have societal impact?

Yes.

Is the study design appropriate and are sufficient details of methods and analysis provided to allow replication by others (Including statistical analysis, if applicable)?

Yes.

Are the conclusions drawn adequately supported by the results?

Yes, overall. The conclusion that resurgent incidence among younger people in Florida is driven by increased social mobility is only weakly supported, though it seems plausible. The more important conclusions about transmission between age classes are better supported.

Does the manuscript cite current literature and discuss limitations?

Yes, though it would be helpful to include more citations.

Is the work clearly and accurately presented (that is, is it well- structured and well-written)?

Yes.

Are the data and code used in the manuscript available?

Data is available. Links are provided in the manuscript.

Have the authors adequately discussed ethical concerns? 
Yes.

\section{Do you recommend this manuscript for publishing?}

Yes.

\section{Detailed Comments}

- Data from state of Florida's reporting of data has been criticized and there is at least one alternative source of data for Florida cases operating. It might be helpful to comment on potential issues with Florida's data.

- Excluded cases before March 1 and under 20 years of age - why?

- "[W]e further relied on a series of county reports" - It would be helpful to provide more detail about these reports and what data they provide.

- Provide more information about Google Mobility Data - how is it collected, and what does it document?

- The description of descriptive analyses in the first section of Methods should be more concrete.

- A SEIR model rather than SIR would be more faithful to the dynamics of COVID-19 infection. It might be helpful to discuss how this difference might affect the results.

- The analysis uses a discrete time SIR model. It would be reasonably simple and more helpful to present the model in discrete time form.

- "A recent review gives a range of serial intervals from 3.1 and 7.5 days" - this should say "to" rather than "and."

- "[A]s derived from our analytic database" - It would be better to be more clear what data.

- This is an interesting way to estimate parameters. Author should document whether this is a novel technique or exists in the literature.

- This paper is complete as is. It would be also interesting to evaluate whether the 20 to 39 class is driving the other two, older, age classes.

- Show restaurant data and Google data for all counties, not just Broward.

- “...jumping to almost 55,000 on May 20 and . " - typo

- "...not the death of death itself." - typo

- Table 1: it is not clear which differences are significant. It would be good to mark significance in the table.

- Figure 10: why Hillsborough and not Broward County? As above, should show all relevant counties including Broward.

- Figure 12: show the other counties 
- The model suggests that transmission among older people only is subcritical. This is a dramatic result, and it might be worth drawing more attention to it. Also, what does the model say about transmission among younger people only? 